УДК 616.8-085.851:616-006

Для цитирования: Тарасова А.В., Куприянова И.Е., Слонимская Е.М., Аксенов М.М., Епанчинцева Е.М., Иванова А.А. Персонализированная терапия онкологических пациентов с нозогенными реакциями. Сибирский вестник психиатрии и наркологии. 2018; 3 (100): 57-61. https://doi.org/10.26617/1810-3111-2018-3(100)-57-61

\title{
Персонализированная терапия онкологических пациентов с нозогенными реакциями
}

\section{Тарасова А.В. ${ }^{1}$, Куприянова И.Е. ${ }^{1}$, Слонимская Е.М. ${ }^{2}$, Аксенов М.М. ${ }^{1}$, Епанчинцева Е.М. ${ }^{1}$, Иванова А.А. ${ }^{1}$}

\author{
${ }^{1}$ Научно-исследовательский институт психического здоровья, Томский национальный исследовательский \\ медицинский иентр Российской академии наук (НИИ психического здоровья Томский НИМЦ) \\ Россия, 634014, Томск, ул. Алеутская, 4 \\ ${ }^{2}$ Научно-исследовательский институт онкологии, Томский национальный исследовательский \\ медиџинский центр Российской академии наук (НИИ онкологии Томский НИМЦ) \\ Россия, 634009. Томск, пер. Кооперативный 5
}

\section{PEЗЮME}

Исследование посвящено вопросам персонализированного психолого-психотерапевтического сопровождения пациентов со злокачественными онкологическими новообразованиями. Материалы и методы. Обследовано 30 пациентов, перенесших лечение по поводу онкопатологии различной локализации. В качестве основных методов обследования использовались клинико-психопатологический, клинико-динамический, психометрический. Результаты. Состояние пациентов при госпитализации характеризовалось преимущественно тревожно-депрессивной симптоматикой различной степени выраженности в рамках невротических расстройств. В лечении на начальном этапе ведущим методом стала фармакотерапия для снятия остроты тревожных проявлений и установления комплаенса между врачом и пациентом. На следующих этапах лечения ведущим методом стала психотерапия. Использовались методы когнитивно-поведенческой терапии, аутотренинг, нейролингвистическое программирование, визуализация, арттерапия. Положительная динамика состояния пациентов отслеживалась как по редукции клинических проявлений, так и по психологическим шкалам. Заключение. Результаты проведенного исследования позволяют судить о преобладании у пациентов тревожно-депрессивной симптоматики в рамках диагностированного расстройства адаптации. Выявлены особенности фармако- и психотерапии пациентов, страдающих онкопатологией. Особенно выраженный положительный эффект отмечался при использовании аутогенных тренировок, а также методов визуализации и арттерапии.

\section{Ключевые слова: рак, нозогенные реакции, психотерапия.}

\section{ВВЕДЕНИЕ}

В последние десятилетия во всем мире неуклонно растет уровень заболеваемости онкологической патологией. По приводимым ВО3 статистическим данным, количество случаев злокачественных новообразований в 2012 г. возросло до 14,1 млн новых случаев (против 12,7 млн в 2008 г.). Количество смертей по причине онкологических заболеваний в 2015 г. увеличилось до 8,8 млн. В связи с этим нервно-психические расстройства, встречающиеся в онкологической практике, приобретают всё большую актуальность. Сам факт выявления онкопатологии, последующая госпитализация в онкологический стационар, а также осложнение или рецидив патологического процесса являются серьезным психотравмирующим событием для любого человека $[5,7]$.

Психические расстройства, формирующиеся в связи с психогенным воздействием онкологического заболевания, определяют как нозогенные реакции. Данные состояния опосредованы категориальными структурами личностных особенностей. Участие личностных расстройств в механизмах трансформации нозогенного воздействия стресса в психические расстройства приводит к типологическому многообразию нозогений и их динамики [6]. Реакции онкопациентов варьируют в широких пределах: от деморализации до диссоциативных расстройств.
По данным зарубежных исследователей, в спектре психических расстройств, коморбидных онкологической патологии, в среднем у $13 \%$ (от 3 до 38\%) больных раком можно диагностировать большой депрессивный эпизод, а более чем у 2/3 (68\%) пациентов выявляются расстройства адаптации различной степени выраженности с преобладанием депрессивной и/или тревожной симптоматики $[8,9$, $10]$.

На современном этапе одним из важнейших направлений психоонкологии является раннее выявление, диагностика и лечение психопатологических расстройств, которые снижают качество жизни онкологических больных и рассматриваются в ряду факторов, ухудшающих клиническое течение, прогноз и выживаемость [2, 4].

Необходимо отметить, что психологические переживания пациентов во многом определяют лечение и исход онкологического заболевания. Эмоциональное состояние играет существенную роль не только в восприимчивости к болезням, включая рак, но и в избавлении от нежелательной патологии. А значит, если человек «психосоматически» может заболеть, то «психосоматически» он может и выздороветь [3, 7, 11]. Поэтому в системном подходе к изучению и лечению рака важно уделить должное внимание психологическим аспектам болезни. 
Эффективность психотерапевтических воздействий в данном случае не может вызывать сомнений. Таким образом, необходимо не только использовать уже имеющиеся техники, но и создавать новые, более эффективные методы психотерапии для их практического воплощения в жизнь [1].

\section{ЦЕЛЬ ИССЛЕДОВАНИЯ}

Изучить методы диагностики и определить прогноз развития нозогенных реакций с учетом личностнохарактерологических особенностей пациентов, страдающих онкопатологией, обеспечить высококвалифицированную медицинскую помощь с включением персонализированного психолого-психотерапевтического сопровождения данных больных.

\section{МАТЕРИАЛ И МЕТОДЫ}

В 2017 г. на базе первого клинического психиатрического отделения ТНИМЦ НИИ психического здоровья проводилось обследование 30 пациентов, страдающих онкологическими заболеваниями различной локализации, в возрасте от 40 до 63 лет с нозогенными реакциями в рамках психических расстройств тревожно-депрессивного спектра (рубрики F41 и F43 по диагностическим критериям МКБ-10).

В качестве основных методов обследования использовались клинико-психопатологический, клинико-динамический, клинико-катамнестический, психометрический. С целью оценки психического состояния пациентов применялись скрининговые шкалы, позволяющие выявить уровни личностной и ситуативной тревоги (шкала Спилбергера-Ханина) и определить тип отношения к имеющемуся заболеванию (опросник ТОБОЛ, Торонтская шкала алекситимии). Диагностика механизмов психологической защиты проводилась на основе опросника Плутчика-Келлермана-Конте. Для учета личностнохарактерологических черт, способных оказывать влияние на формирование и развитие у пациентов нозогенных реакций, использовались методика MMPI и методика Леонгарда-Шмишека для определения типа акцентуации. Для оценки динамики состояния пациентов сравнивались показатели данных шкал на 1-й и 4-й неделях госпитализации.

Проводимые лечебно-профилактические мероприятия условно разделены на три этапа: кризисный, базисный и регредиентный. В исследовании применяли комплексный подход, включая фармако-, психотерапию и психосоциальные воздействия.

\section{РЕЗУЛЬТАТЫ И ОБСУЖДЕНИЕ}

Состояние пациентов при поступлении на госпитализацию характеризовалось преимущественно тревожно-депрессивной симптоматикой различной степени выраженности с агрипническим компонентом и разнообразными психовегетативными проявлениями. Спровоцированное нозогенией ухудшение состояния наблюдалось от 3 до 6 месяцев. По шкале Спилбергера-Ханина ситуативная тревога составляла $45 \pm 3$ балла. В связи с влиянием данных расстройств на качество жизни пациентов нами использовалась шкала работоспособности. Полученные результаты по данной шкале на первом этапе лече- ния указывали на высокие показатели хронической утомляемости, физиологического и когнитивного дискомфорта, также отмечалась низкая мотивация пациентов к трудовой деятельности.

Ведущим методом лечения на кризисном этапе стала психофармакотерапия, так как из-за выраженности тревожных проявлений проведение психотерапевтических мероприятий было затруднено. Дозы психотропных препаратов подбирались с учетом выраженности и длительности психического расстройства, а также индивидуальных особенностей пациентов. По ходу лечения наблюдалась высокая настороженность и тревожность онкопациентов по отношению к применению новых препаратов. Особое внимание обращали на себя пациенты, перенесшие химиотерапию и лучевую терапию. В терапии обследуемых применялись антидепрессанты (рексетин, феварин, леривон) и транквилизаторы (реланиум, феназепам). Пациенты явно отдавали предпочтение более мягким препаратам с минимальным количеством побочных эффектов (атаракс, афобазол). Многие выражали опасения по поводу приема антидепрессантов, с необоснованной уверенностью заявляя о том, что последние им противопоказаны. Учитывая возможные побочные эффекты нейролептиков на систему кроветворения, их применение у онкологических больных невозможно. Таким образом, психотерапевтические мероприятия на данном этапе были направлены на стимуляцию приверженности пациентов к фармакотерапии и установление комплаенса между врачом и пациентом.

После мероприятий по стимуляции приверженности больных к фармакотерапии и по достижении минимального терапевтического эффекта в алгоритм лечения подключались различные психотерапевтические методики. Персонализированное психолого-психотерапевтическое сопровождение проводилось либо в индивидуализированном формате, либо в группах. Пациенты с выраженными личностными особенностями требовали к себе повышенного внимания терапевта. Они некурабельны или трудно курабельны в групповой работе, в связи с чем им показана индивидуальная психотерапия. В качестве основных использовались методы когнитивноповеденческой терапии, аутотренинг, нейролингвистическое программирование, визуализация, арттерапия.

Пациенты с тревожными проявлениями, ориентированные на лечение, демонстрировали хороший ответ на проводимые психотерапевтические мероприятия. Выраженный эффект наблюдался при использовании аутогенных тренировок с последующей визуализацией. Ввиду концентрации тревожных и тягостных мыслей по поводу имеющейся онкопатологии пациенты визуализировали желаемое улучшение соматического состояния, уменьшение размеров и агрессивности опухоли, усиление собственных сил организма, повышая, таким образом, мотивацию и настрой для дальнейшей борьбы с заболеванием и, как следствие, снижая страх перед ним. 
Также следует подчеркнуть усиление положительной динамики на фоне присоединения методов арттерапии, хотя пациенты на начальном этапе включались в неё неохотно. Но, отмечая субъективное улучшение с каждым сеансом, с энтузиазмом продолжали выполнять задания и даже вносить собственные предложения и темы для обсуждения и проработки. Таким образом, в ходе этапа базисного лечения с акцентом на психотерапевтический комплекс наблюдалась положительная динамика: снижение уровня тревожно-фобической симптоматики, выравнивание фона настроения, редукция выраженных психовегетативных проявлений, нормализация сна и восстановление режима «сон - бодрствование». Вместе с тем были выявлены пациенты и с неполным ответом на терапию, что обусловлено особенностями их личности $(10 \%)$.

На регредиентном этапе лечения положительная динамика состояния пациентов отслеживалась по редукции клинических проявлений и по психологическим шкалам: показатели шкалы СпилбергераХанина ситуативной тревоги значительно снизились (с $45 \pm 3$ балла до $25 \pm 5$ баллов); изменения показателей, оценивающих уровень личностной тревоги, оказались незначительными. Результаты по шкале работоспособности на 4-й неделе лечения указывали на значительное снижение хронической утомляемости, ликвидацию физиологического и когнитивного дискомфорта, а также повышение мотивации пациентов к трудовой деятельности. В период завершения стационарного лечения основной акцент ставился на психосоциотерапевтические мероприятия, пациент подготавливался к этапу поддерживающей терапии, осуществляемой после выписки. Кроме того, на данном этапе активно использовалось психообразование в группах с целью повышения и выработки саногенных установок пациентов.

\section{ЗАКЛЮЧЕНИЕ}

Результаты проведенного исследования позволяют судить о преобладании расстройств тревожнодепрессивного круга у пациентов с нозогенными реакциями. По данным различных исследователей, такой тип реактивных состояний распространен не только в онкологической практике, но и отмечается при других соматических заболеваниях. Особенностью ведения пациентов, страдающих онкопатологией, являлась их высокая настороженность и тревожность по отношению к применению психотропных препаратов, что требует активных психотерапевтических мероприятий, направленных на стимуляцию приверженности пациентов к фармакотерапии и установление комплаентности между врачом и пациентом. На фоне комплексной психотерапии пациенты, ориентированные на лечение, демонстрировали положительный эффект в виде редукции клинических проявлений и повышения саногенных установок. Наиболее выраженный положительный эффект продемонстрирован при использовании аутогенных тренировок, а также методов визуализации и арттерапии.
Кроме того, была выделена группа пациентов $(10 \%)$ с неполным ответом на терапию, что обусловлено особенностями их личности. Данная категория пациентов требует пристального внимания и наблюдения в динамике ввиду более неблагоприятного прогноза психических расстройств.

В связи с высокой распространенностью онкопатологии среди населения необходимо создание образовательных групп для онкологических пациентов. В образовательные программы следует включить информативные разделы, посвященные способам поведения в стрессовой ситуации тяжелого заболевания, здоровому образу жизни и психогигиене.

\section{КОНФЛИКТ ИНТЕРЕСОВ}

Авторы декларируют отсутствие явных и потенциальных конфликтов интересов в связи с публикацией данной статьи.

\section{ИСТОЧНИК ФИНАНСИРОВАНИЯ}

Статья подготовлена в рамках поисковых научных исследований «Технологии индивидуализированной многоуровневой дифференцированной реабилитации больных с непсихотическими психическими расстройствами», «Персонализированное психолого-психотерапевтическое сопровождение больных с нозогенными реакциями». Шифр темы 0550-2016-0403. Номер госрегистрации АААА-А16116042610052-0.

\section{СООТВЕТСТВИЕ ПРИНЦИПАМ ЭТИКИ}

Исследование с участием людей проведено с соблюдением принципов Хельсинкской декларации BMА и одобрено Локальным этическим комитетом при НИИ психического здоровья Томского НИМЦ (протокол № 98 от 20 марта 2017 г.).

\section{ЛИТЕРАТУРА}

1. Бескова Д.А., Сиранчиева О.А. Дифференцированный подход к выбору метода психотерапии нозогенных реакций на госпитальном этапе лечения онкологических заболеваний. Психические расстройства в общей медицине. 2009; 1: 4652.

2. Дубский С.В., Куприянова И.Е., Чойнзонов Е.Л., Балацкая Л.Н. Психологическая реабилитация и оценка качества жизни больных раком щитовидной железы. Сибирский онкологический журнал. 2008; 4: 17-21.

3. Саймонтон К., Саймонтон С. Психотерапия рака. СПб.: Питер, 2001: 288.

4. Самушия М.А. Психические расстройства у пациенток со злокачественными опухолями органов женской репродуктивной системы (обзор литературы). Психические расстройства в общей медиџине. 2010; 4: 24-31.

5. Семке В.Я., Чойнзонов Е.Ц., Куприянова И.Е., Балацкая Л.Н. Развитие сибирской психоонкологии. Томск: Изд-во ТГУ, 2008: 198

6. Смулевич А.Б., Иванов С.В., Самушия М.А. Патохарактерологическое предрасположение и формирование нозогенных (провоцированных соматическим заболеванием) психических расстройств. Психические расстройства в общей медицине. 2014; 2: 7-13.

7. Чулкова В.А., Пестерева Е.В. Клинико-психологические аспекты в онкологии. Вестник Санкт-Петербургского государственного университета. 2010; 1: 91-100.

8. Kissane D.W., Maj M., Sartorius N. Depression and cancer. John Wiley \& Sons, Ltd, 2011: 244.

9. Mehnert A., Koch U. Prevalence of acute and post-traumatic stress disorder and comorbid mental disorders in breast cancer patients during primary cancer care: a prospective study. Psychooncology. 2007; 16 (3): 181-8. 
10. Raison C.L., Miller A.H. Depression in cancer: mechanisms and disease progression. Biol Psychiat. 2003; 54: 283-94.

Поступила в редакцию 9.04.2018

Roy-Byrne P.P., Davidso K.W., Kessler R.C. et al. Anxiety Disor-

Утверждена к печати 25.06.2018 ders and Comorbid Medical Illness. Focus. 2008; 6: 467-85.

Тарасова Алена Владимировна, младший научный сотрудник отделения пограничных состояний.

Куприянова Ирина Евгеньевна, доктор медицинских наук, профессор, ведущий научный сотрудник отделения пограничных состояний. Слонимская Елена Михаловна, доктор медицинских наук, профессор, руководитель отделения общей онкологии.

Аксенов Михаил Михайлович, доктор медицинских наук, профессор, заведующий отделением пограничных состояний.

Епанчинцева Елена Макаровна, кандидат медицинских наук, заведующая первым клиническим психиатрическим отделением.

Иванова Алла Алимомедовна, кандидат психологических наук, медицинский психолог консультативно-диагностического отделения.

Куприянова Ирина Евгеньевна, irinakupr@rambler.ru

For citation: Tarasova A.V., Kupriyanova I.E., Slonimskaya E.M., Axenov M.M., Epanchintseva E.M., Ivanova A.A. A personalized therapy of nosogenic reactions in cancer patients. Siberian Herald of Psychiatry and Addiction Psychiatry. 2018; 3 (100): 5761. https://doi.org/10.26617/1810-3111-2018-3(100)-57-61

\title{
A personalized therapy of nosogenic reactions in cancer patients Tarasova A.V. ${ }^{1}$, Kupriyanova I.E. ${ }^{1}$, Slonimskaya E.M. ${ }^{2}$, Axenov M.M. ${ }^{1}$, Epanchintseva E.M. ${ }^{1}$, Ivanova A.A. ${ }^{1}$
}

\author{
${ }^{1}$ Mental Health Research Institute, Tomsk National Research Medical Center, Russian Academy of Sciences \\ Aleutskaya Street 4, 634014, Tomsk, Russian Federation \\ ${ }^{2}$ Oncology Research Institute, Tomsk National Research Medical Center, Russian Academy of Sciences \\ Kooperativny 5, 634009, Tomsk, Russian Federation
}

\section{ABSTRACT}

The study was devoted to the problems of a personalized psychological and psychotherapeutic care for cancer patients. Materials and Methods. 30 persons who had a cancer therapy were examined. The main methods of examination were psychopathologic, dynamic, psychometric. Results. At admission the status of patients was characterized mostly by anxiety-depressive symptoms of various degree. These patients were diagnosed as having borderline mental disorders. In the start of treatment leading method was pharmacotherapy for anxiety reduction and compliance setting between doctor and patient. At the next stages of treatment the leading method was psychotherapy. Methods of cognitive-behavioral therapy, auto-training, neuro-linguistic programming, visualization and art-therapy were used. Positive dynamics of patients condition was monitored both by reduction of clinical manifestations and by psychological scales. Conclusion. Results of the study allowed to judge prevalence of anxietydepressive disorders in patients with nosogenic reactions. Features of pharmaco- and psychotherapy in cancer patients were revealed. Use of auto-training, visualization and art-therapy exerted more expressed positive effect.

Keywords: cancer, nosogenic reactions, psychotherapy.

\section{REFERENCES}

1. Beskova D.A., Siranchiyeva O.A. Differencirovannyj podhod k vyboru metoda psihoterapii nozogennyh reakcij na gospital'nom jetape lechenija onkologicheskih zabolevanij [The differentiated approach to the choice of a method of a psychotherapy the nozogenic reactions at the hospital stage of cancer treatment]. Psihicheskie rasstrojstva $v$ obshhej medicine - Mental Disorders in General Medicine. 2009; 1: 46-52 (in Russian).

2. Dubsky S.V., Kupriyanova I.E., Choynzonov E.L., Balatskaya L.N. Psihologicheskaja reabilitacija i ocenka kachestva zhizni bol'nyh rakom shhitovidnoj zhelezy [Psychological after treatment and assessment of quality of life of patients with cancer of a thyroid gland]. Sibirskij onkologicheskij zhurnal - Siberian Oncologic Journal. 2008; 4: 17-21 (in Russian).

3. Saymonton K., Saymonton S. Psihoterapija raka [Psychotherapy of cancer]. Saint-Petersburg: "Piter", 2001: 288 (in Russian).

4. Samushiya M.A. Psihicheskie rasstrojstva u pacientok so zlokachestvennymi opuholjami organov zhenskoj reproduktivnoj sistemy (obzor literatury) [Mental disorders in patients with female reproductive system malignancies (a review of literature)]. Psihicheskie rasstrojstva v obshhej medicine - Mental Disorders in General Medicine. 2010; 4: 24-31 (in Russian).

5. Semke V.Ya., Choynzonov E.Ts., Kupriyanova I.E., Balatskaya L.N. Razvitie sibirskoj psihoonkologii [Development of the Siberian psychooncology]. Tomsk: Publishing House of Tomsk State University, 2008: 198 (in Russian).
6. Smulevich A.B., Ivanov S.V., Samushiya M.A. Patoharakterologicheskoe predraspolozhenie $\mathrm{i}$ formirovanie nozogennyh (provocirovannyh somaticheskim zabolevaniem) psihicheskih rasstrojstv.[Personality predisposition and mechanism of occurrence nozogenic (provoked by a somatic disease) mental disorders]. Psihicheskie rasstrojstva $v$ obshhej medicine - Mental Disorders in General Medicine. 2014; 2: 7 -13 (in Russian).

7. Chulkova V.A., Pestereva E.V. Kliniko-psihologicheskie aspekty $\mathrm{v}$ onkologii [Clinical and psychological aspects in oncology]. Vestnik Sankt-Peterburgskogo gosudarstvennogo universiteta Bulletin of St. Petersburg State University. 2010; 1: 91-100 (in Russian).

8. Kissane D.W., Maj M., Sartorius N. Depression and cancer. John Wiley \& Sons, Ltd, 2011: 244.

9. Mehnert A., Koch U. Prevalence of acute and post-traumatic stress disorder and comorbid mental disorders in breast cancer patients during primary cancer care: a prospective study. Psychooncology. 2007; 16 (3): 181-8.

10. Raison C.L., Miller A.H. Depression in cancer: mechanisms and disease progression. Biol Psychiat. 2003; 54: 283-94.

11. Roy-Byrne P.P., Davidso K.W., Kessler R.C. et al. Anxiety Disorders and Comorbid Medical Illness. Focus. 2008; 6: 467-85.

Received April 9.2017 Accepted June 25.2018 
Tarasova Alyona V., junior researcher of Borderline States Department, Mental Health Research Institute, Tomsk National Research Medical Center, Russian Academy of Sciences, Tomsk, Russian Federation.

Kupriyanova Irina E., MD, Prof., senior researcher of Borderline States Department, Mental Health Research Institute, Tomsk National Research Medical Center, Russian Academy of Sciences, Tomsk, Russian Federation.

Slonimskaya Elena M. MD, Prof., Head of General Oncology Department, Oncology Research Institute, Tomsk National Research Medical Center, Russian Academy of Sciences, Tomsk, Russian Federation.

Axenov Mikhail M., MD, Prof., of Borderline States Department, Mental Health Research Institute, Tomsk National Research Medical Center, Russian Academy of Sciences, Tomsk, Russian Federation.

Epanchintseva Elena M., PhD, Head of the First Clinical Unit, Mental Health Research Institute, Tomsk National Research Medical Center, Russian Academy of Sciences, Tomsk, Russian Federation.

Ivanova Alla A., PhD for Psychology, medical psychologist of Consultative-Diagnostic Unit, Mental Health Research Institute, Tomsk National Research Medical Center, Russian Academy of Sciences, Tomsk, Russian Federation.

Kupriyanova Irina E., irinakupr@rambler.ru 